\title{
Entropy
}

ISSN 1099-4300

www.mdpi.org/entropy/

\section{Effect of Magnetic Field on Entropy Generation Due to Laminar Forced Convection Past a Horizontal Flat Plate}

\author{
Mohammed Q. Al-Odat ${ }^{1}$, Renhe A. Damseh ${ }^{2}$ and Moh'd A. Al-Nimr ${ }^{3}$
}

${ }^{1}$ Mechanical Engineering Department, Faculty of Engineering Technology

Al-Balqa Applied University, P.O. Box 340558 Amman 11134 Jordan

Tel: +9626-4892345; Fax: +9626-4892345; Email: m_alodat@hotmail.com

${ }^{2}$ Mechanical Engineering Department, Al-Husn University College

Al-Balqa Applied University, Irbid-Jordan
${ }^{3}$ Mechanical Engineering Department, Jordan University of Science and Technology, Irbid-Jordan

Received: 9 February 2004 / Accepted: 5 June 2004 / Published: 5 June 2004

\begin{abstract}
Magnetic field effect on local entropy generation due to steady two-dimensional laminar forced convection flow past a horizontal plate was numerically investigated. This study was focused on the entropy generation characteristics and its dependency on various dimensionless parameters. The effect of various dimensionless parameters, such as Hartmann number $(\mathrm{Ha})$, Eckert number $(E c)$, Prandtl number $(P r)$, Joule heating parameter $(R)$ and the free stream temperature parameter $\left(\theta_{\infty}\right)$ on the entropy generation characteristics is analyzed. The dimensionless governing equations in Cartesian coordinate were solved by an implicit finite difference technique. The solutions were carried out for $\mathrm{Ha}^{2}=0.5-3, E c=0.01-0.05, \mathrm{Pr}=1-5$ and $\theta_{\infty}=1.1-2.5$. It was found that, the entropy generation increased with increasing $H a, E c$ and $R$. While, increasing the free stream temperature parameter, and Prandtl number tend to decrease the local entropy generation.
\end{abstract}

Keywords: Forced convection, Magnetic field, Joule heating, Entropy generation 


\section{Introduction}

The optimal design of thermal systems can be achieved by minimizing entropy generation in the systems. This issue has been the topic of great importance in many engineering field such as heat exchangers, cooling of nuclear reactors, MHD power generators, geophysical fluid dynamics, energy storage systems and cooling of electronic devices, etc. Entropy generation is associated with thermodynamics irreversibility, which is common in all types of heat transfer processes. Different sources of irreversibility are responsible for entropy generation such as heat transfer across finite temperature gradient, characteristics of convective heat transfer, magnetic field effect, viscous dissipation effect etc. Entropy generation in thermal engineering systems destroys system available work and thus reduces its efficiency. Bejan $[1,2]$ illustrated that the flow parameter could be selected in order to minimizes the irreversibility associated with a specific convective heat transfer processes. Abu-Hijleh and Heilen [3] investigated entropy generation due to laminar mixed convection from an isothermal rotating cylinder. This study indicated that entropy generation is increased as the Reynolds number and the buoyancy parameter increased. Tasnim et al. [4] presented an analytical work to study the first and second laws (of thermodynamics) characteristics of flow and heat transfer inside a vertical channel made of two parallel plates embedded in a porous medium and under the action of transverse magnetic field. They demonstrated that the group parameter have a significant effect on entropy generation such that the higher values of group parameters cause higher entropy generation. Mahmud and Fraser [5] analyzed the second law (of thermodynamics) characteristics of heat and fluid flow due to forced convection inside a channel with circular cross-section and channel made of two parallel plates. They derived an analytical expression for the entropy generation and Bejan number. Second law analysis of combined heat and mass transfer in internal and external flows was presented by Carrington and Sun [6]. They derived an equation for the entropy generation and discussed the applicability of the resulting equation. Arpaci and Selamet [7] investigated the entropy production in boundary layers. They showed that the entropy generation for forced convective heat transfer is due to temperature gradient and viscosity effect in the fluid. Khalkhali et al. [8] developed a thermodynamic model of conventional cylindrical heat pipes based on the second law of thermodynamics. Their result showed that entropy generation is caused by the temperature difference between the hot and cold reservoirs, the frictional losses in the working fluid flows, and the vapor temperature/pressure drop along the heat pipe. Abu-Hijleh [9] computed entropy generation due to laminar mixed heat convection from an isothermal heated cylinder in an air cross flow for different values of the Reynolds number, buoyancy parameter, and cylinder diameter. His results showed that large cylinder diameters resulted in lower entropy generation. Mahmud and Fraser [10] investigated analytically the effect of radiation heat transfer on mixed convection through a vertical channel in the presence of transverse magnetic field, applying both the first and second law to analyze the problem. They found that the radiation and mixed convection parameters have dominating influence on entropy generation rate than Hartmann number. Haddad et al. [11] considered the local entropy generation of steady two- 
dimensional symmetric flow past a parabolic cylinder in a uniform stream parallel to its axis. Their results indicated that the thermal entropy generation increased as the temperature difference increased, while the viscous entropy generation decreased as Reynolds number increased. Buhler [12] analyzed the flow of an incompressible, viscous electrically conducting fluid in magnetic field with and without buoyancy effect. Raptis and Kafoussias [13] studied the flow and heat transfer characteristics in the presence of porous medium and magnetic field. Chamkha [14] studied the problem of steady, laminar, free convection flows over vertical porous surface in the presence of magnetic filed and heat generation or absorption. Elbashbeshy [15] investigated heat transfer over a stretching surface with variable and uniform heat flux subjected to suction. The common outcome of references [12-15] is that the presence of magnetic field tends to slowdown the fluid motion and thus increases the fluid temperature.

The above-mentioned investigations can be classified into two main categories. The first one deals with the effect of magnetic field on the first law (of thermodynamics) characteristics of heat transfer and fluid flow in external or internal flow processes. Whereas, the second category deals just with the second law (of thermodynamics) characteristics of heat transfer and fluid flow in internal flows with magnetic field effect, or in external flow without magnetic field effect. Based on the above brief literature review, there have been no reported investigations, which clarify the magnetic filed effects on the second law (of thermodynamics) characteristics of convective heat transfer in external flow processes. This constitutes the motive of the present study. Therefore, the main objective of this article is to investigate the effect of transverse magnetic field on local entropy generation due to steady twodimensional laminar forced convection flow past a horizontal plate. In the present work, the full Navier-Stockes equations are solved using an implicit finite difference method to describe laminar forced convection over an isothermal flat plate. The entropy generation rates due to forced convection about a flat plate are computed for different values of Hartmann number $(H a)$, Eckert number $(E c)$, Prandtl number $(P r)$, Joule heating parameter $(R)$ and the temperature difference between the flat plate and the free-stream $\left(\theta_{\infty}\right)$.

\section{Problem formulation}

Consider the two-dimensional steady, laminar MHD forced convection flow of an electrically conducting, incompressible, Newtonian fluid over an isothermal plate in the presence of a transverse magnetic field $\left(B_{y}\right)$ applied normal to the flow direction. A schematic diagram of the problem under consideration is shown in Fig. 1. The fluid is assumed to be incompressible with constant properties. The non-dimensional equations for steady state two-dimensional laminar MHD force convection over a flat plate are given by [16]

$$
\frac{\partial U}{\partial X}+\frac{\partial V}{\partial Y}=0
$$




$$
\begin{aligned}
& U \frac{\partial U}{\partial X}+V \frac{\partial U}{\partial Y}=\frac{\partial^{2} U}{\partial Y^{2}}-H a^{2} U \\
& U \frac{\partial \theta}{\partial X}+V \frac{\partial \theta}{\partial Y}=\frac{1}{\operatorname{Pr}} \frac{\partial^{2} \theta}{\partial Y^{2}}+E c\left(\frac{\partial U}{\partial Y}\right)^{2}+R U^{2}
\end{aligned}
$$

The dimensionless parameters are defined as:

$$
\left.\begin{array}{l}
\theta=\frac{T-T_{\infty}}{T_{w}-T_{\infty}}, \quad X=\frac{x}{x_{o}}, \quad Y=\frac{y}{x_{o}} \quad V=\frac{v}{u_{\infty}}, \quad U=\frac{u}{u_{\infty}}, \quad x_{0}=\frac{v}{u_{\infty}}, \\
R=\frac{\sigma B_{y}^{2} \mu}{\rho^{2} C_{p} \Delta T}, \quad E c=\frac{U_{\infty}^{2}}{C_{p} \Delta T}, \quad H a^{2}=\frac{\sigma B_{y}^{2} v}{u_{\infty}^{2} \rho}, \quad \Delta T=\left(T_{w}-T_{\infty}\right), \quad \operatorname{Pr}=\frac{v}{\alpha}, \quad \theta_{\infty}=\frac{T_{\infty}}{\Delta T}
\end{array}\right\}
$$

Where is $(X, Y)$ are the Cartesian coordinates along and normal to the plate, respectively, and $(U, V)$ are the dimensionless velocity components along $X$ and $Y$, respectively. $\theta$ is the dimensionless temperature, $x_{o}$ is the characteristic length of the plate, $\left(\theta_{\infty}\right)$ is free stream temperature parameter. $H a$, $P r, E c$ and $R$ are Prandtl number, Eckert number, Hartmann number, and the Joule heating parameter, respectively. $u_{\infty}$ is the velocity of the potential flow outside the boundary layer.

The physical problem assumes the following dimensionless boundary conditions

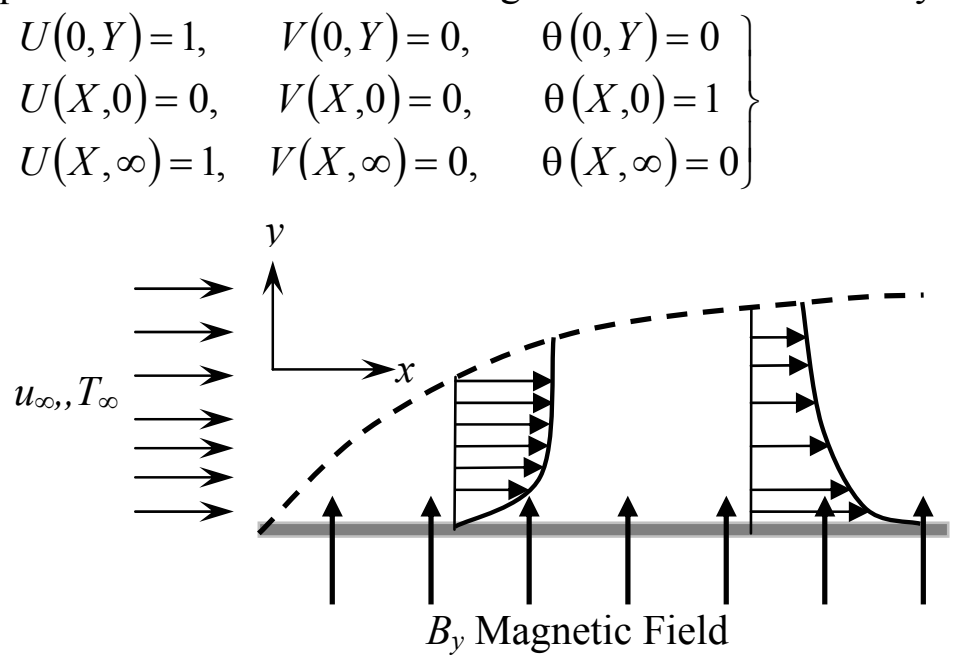

Figure1. Schematic diagram of the problem under consideration.

\section{Entropy generation}

Assuming that the fluid is Newtonian and incompressible, and if it obeys the Fourier law of heat conduction, the non-dimensional form of the volumetric rate of local entropy generation in 2-D Cartesian coordinates is [17]:

$$
S^{\prime \prime \prime}=\frac{1}{\theta+\theta_{\infty}}\left(\frac{\partial U}{\partial Y}\right)^{2}+\frac{1}{\operatorname{Pr} E c\left(\theta+\theta_{\infty}\right)^{2}}\left[\left(\frac{\partial \theta}{\partial X}\right)^{2}+\left(\frac{\partial \theta}{\partial Y}\right)^{2}\right]+\frac{1}{\theta+\theta_{\infty}} \frac{R U^{2}}{E c}
$$

The dimensionless volumetric entropy generation is defined as: 


$$
S^{\prime \prime \prime}=\frac{s^{\prime \prime \prime}}{s_{o}^{\prime \prime \prime}} \text { where } s_{o}^{\prime \prime \prime} \text { is given by } s_{o}^{\prime \prime \prime}=\frac{u_{\infty}^{4} \mu}{v^{2} \Delta T} \text {. }
$$

The entropy generation equation consists of three parts, the first part is the irreversibility due to finite temperature gradient and generally termed as heat transfer irreversibility, this part is due to conduction, and the second is due to viscous dissipation while the third is due to Joule heating. Entropy generation is computed after the numerical solution of the velocity and temperature distributions has been obtained.

\section{Solution Methodology}

The governing differential equations (1)-(3) along with the boundary conditions Eq. (4) were solved numerically using an implicit finite difference method similar to that described by Patanker [18]. Applying central differences for spatial derivatives in the governing equations, a nonlinear system of equations is generated over a non-uniform grid, to accommodate the steep velocity and temperature at the wall. Non-uniform grid spacing was used in the $X$-direction with most grids located near the wall and uniform grid was adopted in the $Y$-direction. Constant step sizes of 0.01 were used in the normal direction $(Y)$ and variable step sizes in longitudinal direction $(X)$ with initial step size of 0.001 and a growth factor of 1.002. Due to nonlinearities of the governing equations, an iterative solution technique is required to solve the resulting system of nonlinear algebraic equations, in this study Gauss-Seidel iterative procedure associated with the relaxation parameters was considered. The computational domain was restricted to finite dimensions. Here, the height of the plate $X_{\max }$ assumed to be 15. The boundary layer thickness $Y_{\max }$ was taken as 4 . The maximum value of $Y$ was chosen as 4 after some preliminary investigations so that, the last two boundary conditions (5) are satisfied (i.e. $U \rightarrow 0, \theta \rightarrow 0$ as $Y \rightarrow \infty$ ). Underrelaxation is required to secure convergence of the iteration procedure. The range for the Underrelaxation factor is taken as 0.1-0.6 for the velocity and temperature fields. The convergence criteria in iteration is stated as

$$
\left|\frac{\phi_{\text {new }}-\phi_{\text {old }}}{\phi_{\text {new }}}\right| \leq \varepsilon
$$

where $\phi_{\text {new }}$ and $\phi_{\text {old }}$ denote one of the main variables $U, V$, and $\theta$ and the subscripts new and old denote the values corresponding to the new iteration and old iteration. The value for the tolerance $\varepsilon$ is taken as $10^{-6}$.

A grid independence study was carried out with $41 \times 41,61 \times 61,81 \times 81$ mesh size. The results obtained using a finer grid of $81 \times 81$ do not reveal discernible changes in the predicted heat transfer and flow field. Thus, due to computational cost and accuracy considerations a $61 \times 61$-mesh size was used in this investigation.

In order to assess the accuracy of presented numerical technique, the results obtained by the present method are compared of the classical forced-convection problem past an impermeable flat plate that 
reported by Kays and Crawford [19]. Tables 1 and 2 illustrate a comparison for the dimensionless velocity values, and the temperature gradient at the wall $\theta^{\prime}(0)$ of the present study in absence of the magnetic field effect and the viscous dissipation (i.e. $H a^{2}=R=E c=0$ ) with those of ref. [19]. It can be seen that the present results agree very well with the previously published data. This has established confidence in the numerical results presented in this paper.

Table 1. Comparison of the dimensionless velocity $U$ distribution.

\begin{tabular}{|c|c|c|}
\hline$\eta=\frac{Y}{\sqrt{X}}$ & $\begin{array}{c}u=\frac{u}{u_{\infty}}=f^{\prime}(\eta), \\
\text { Kays and Crawford } \\
{[19]}\end{array}$ & $\begin{array}{c}U=\frac{u}{u_{\infty}}, \\
\text { (Present results) }\end{array}$ \\
\hline \hline 0 & 0 & 0 \\
\hline 0.4 & 0.133 & 0.131 \\
\hline 0.8 & 0.265 & 0.272 \\
\hline 1.2 & 0.394 & 0.402 \\
\hline 1.6 & 0.517 & 0.519 \\
\hline 2.0 & 0.630 & 0.632 \\
\hline 2.8 & 0.812 & 0.816 \\
\hline 3.6 & 0.923 & 0.925 \\
\hline 4.4 & 0.979 & 0.980 \\
\hline 4.6 & ----- & 0.994 \\
\hline
\end{tabular}

Table 2. Comparison of the wall temperature gradient $\theta^{\prime}(0)$ for various values of $\operatorname{Pr}$.

\begin{tabular}{|c|c|c|}
\hline \multirow{2}{*}{$P r$} & $\theta^{\prime}(0)$, & $\frac{\partial \theta^{\prime}}{\partial Y},($ Present results $)$ \\
\hline 0.5 & Kays and Crawford [19] & 0.261 \\
\hline 0.7 & 0.259 & 0.290 \\
\hline 1.0 & 0.292 & 0.331 \\
\hline 7.0 & 0.332 & 0.643 \\
\hline 10.0 & 0.645 & 0.729 \\
\hline 17.0 & 0.730 & 0.838 \\
\hline
\end{tabular}

\section{Results and discussion}

Figure 2 shows the dimensionless velocity distributions at different values of Hartmann number $(\mathrm{Ha})$. It is clear that, increase the value of $(\mathrm{Ha})$ have a tendency to slow down the fluid motion. This is because the presence of the transverse magnetic field creates a resistive force similar to the drag force 
that acts in the opposite direction of the fluid motion, thus causing the velocity of the fluid to decrease. As expected, increasing $(\mathrm{Ha})$ causes the fluid to become warmer and therefore increase its temperature as shown in Fig. 3. This behavior is attributed to decrease of the fluid velocity due to the magnetic field.

The effect of Hartmann number $(\mathrm{Ha})$ on the dimensionless volumetric entropy generation profiles is displayed in Fig.4. This figure shows that the entropy generation is slightly increases with Hartmann number. The Hartmann number is not too much dominating on entropy generation. A large variation of ( $\mathrm{Ha}$ ) causes a small variation in the rate of entropy generation.

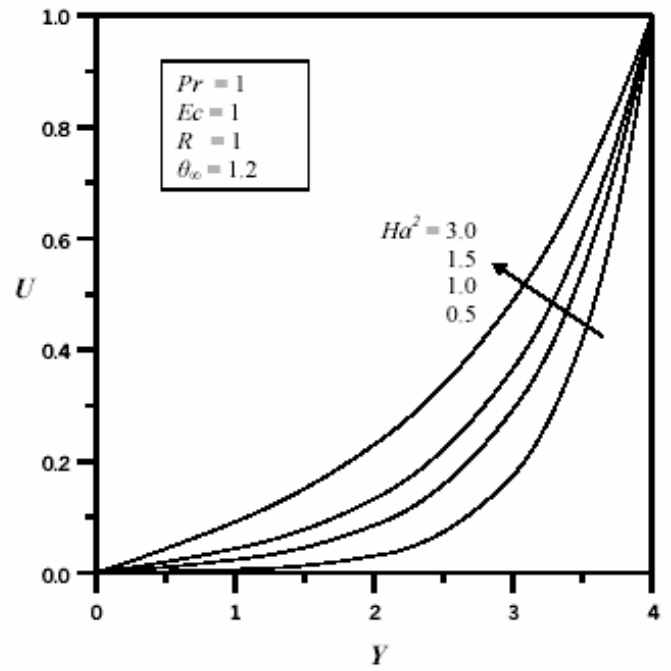

Figure 2. Dimensionless velocity distribution for different values of Hartmann number $(\mathrm{Ha})$ at the midpoint length of the plate $(X=7.5)$.

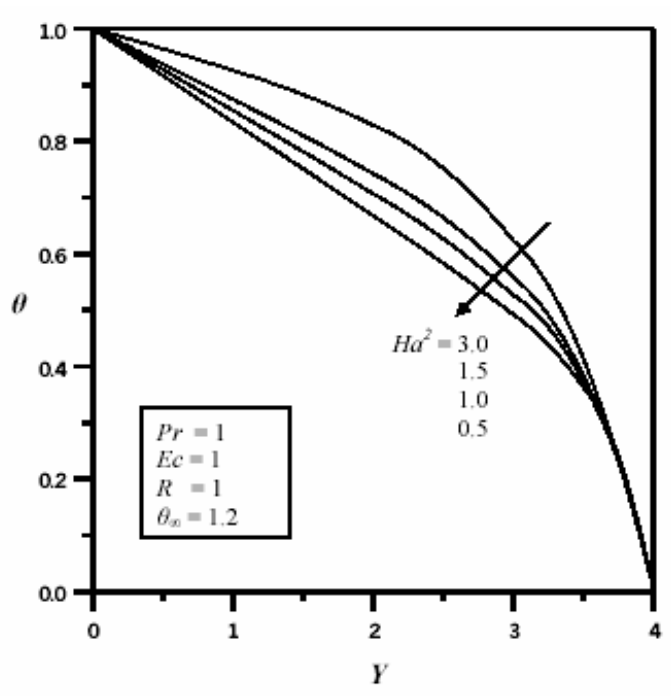

Figure 3. Dimensionless temperature distribution for different values of Hartmann number $(\mathrm{Ha})$ at the midpoint length of the plate $(X=7.5)$.

Figure 5 illustrates the effect of the Joule heating parameter $(R)$ on the dimensionless volumetric entropy generation distributions with $Y$. From this figure, it can be seen that the local entropy generation increases with $R$. This behavior may be explained by the fact that the energy loss (exergy destruction) increases with the Joule heating parameter.

The influence of Prandtl number $(P r)$ on the dimensionless volumetric entropy generation distributions with $Y$ is plotted in Fig. 6. As Prandtl number $P r$ increases the dimensionless volumetric entropy generation distributions decreases. This due to decrease in the temperature gradient with Prandtl number.

Figure 7 shows the influence of Eckert number $(E c)$ on the dimensionless volumetric entropy generation distributions with $Y$. It is obvious that the entropy generation increases significantly with Eckert number. This behavior is attributed to the increase of viscous dissipation as $E c$ increase.

The effect of the free stream temperature parameter $\left(\theta_{\infty}\right)$ on the dimensionless volumetric entropy generation distributions with $Y$ is plotted in Fig. 8. As expected, the volumetric entropy generation decreases as the free stream temperature parameter increases. This can be explained by recalling the 
definition of $\theta_{\infty}=\frac{T_{\infty}}{\Delta T}$; increasing $\theta_{\infty}$ could be achieved by decreasing $\Delta T$ or increasing the free stream temperature $T_{\infty}$. Decreasing $\Delta T$ tends to decrease heat transfer rate and thus the irreversibilities associated with the heat transfer process. Furthermore, increasing the free stream temperature means a reduction in the temperature difference between the free stream and the plate because the plate temperature is always higher than the free stream temperature

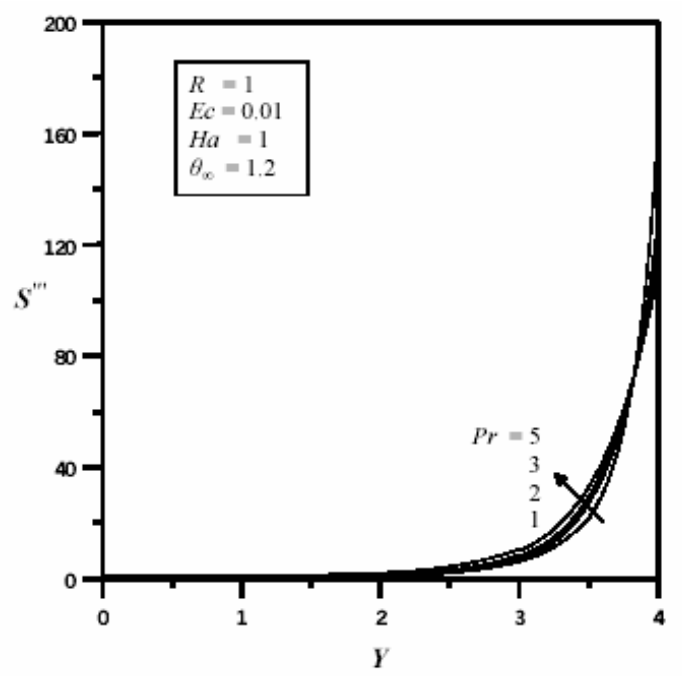

Figure 4. Dimensionless entropy generation for different values of Hartmann number $(\mathrm{Ha})$ at the end edge of the plate $(X=15)$.

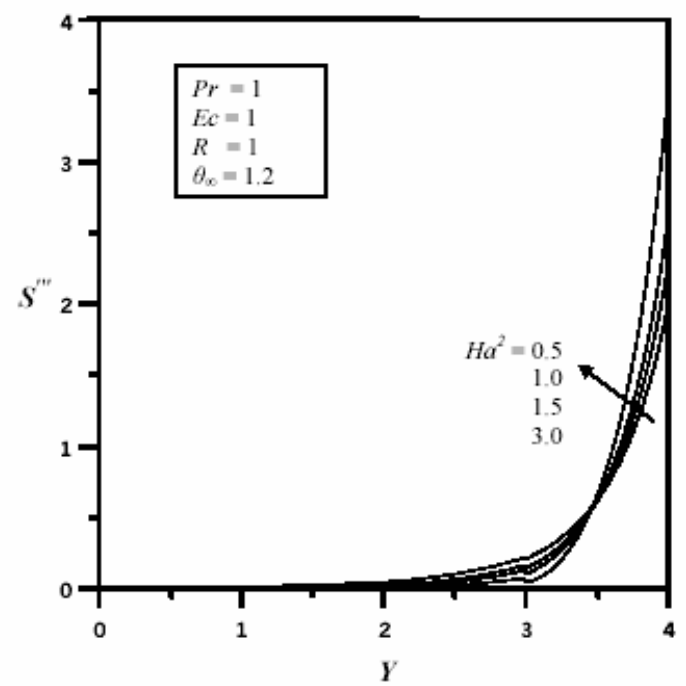

Figure 6. Dimensionless entropy generation for different values of Prandtl numbers $(P r)$ at the end edge of the plate $(X=15)$.

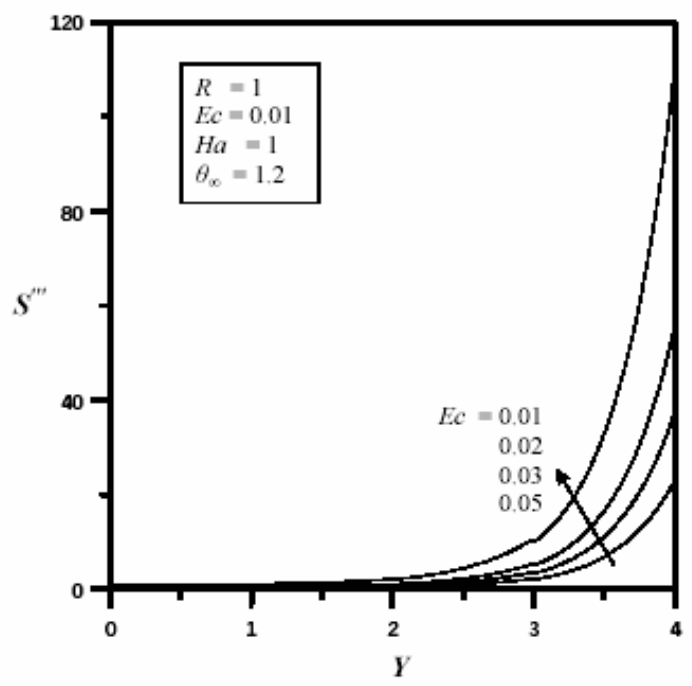

Figure 5. Dimensionless entropy generation for different values of Joule heating factor $(R)$ at the end edge of the plate $(X=15)$.

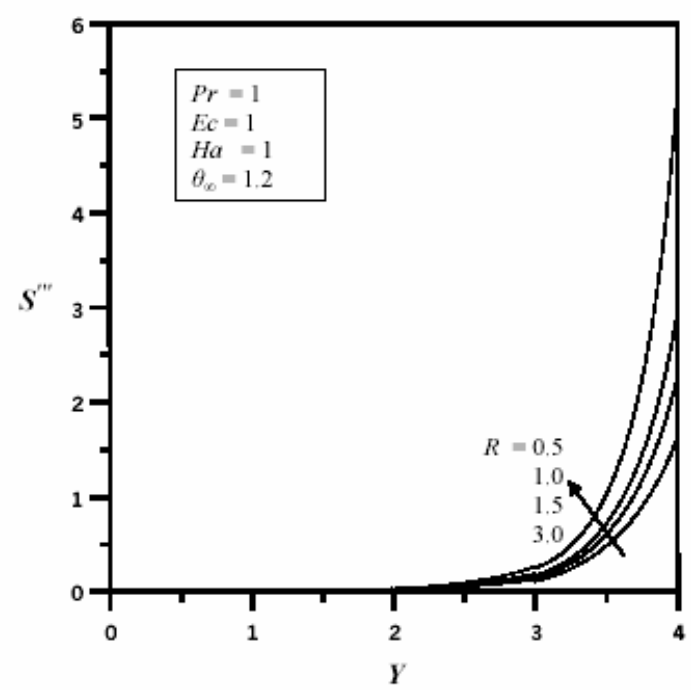

Figure 7. Dimensionless entropy generation for different values of Eckert numbers $(E c)$ at the end edge of the plate $(X=15)$. 


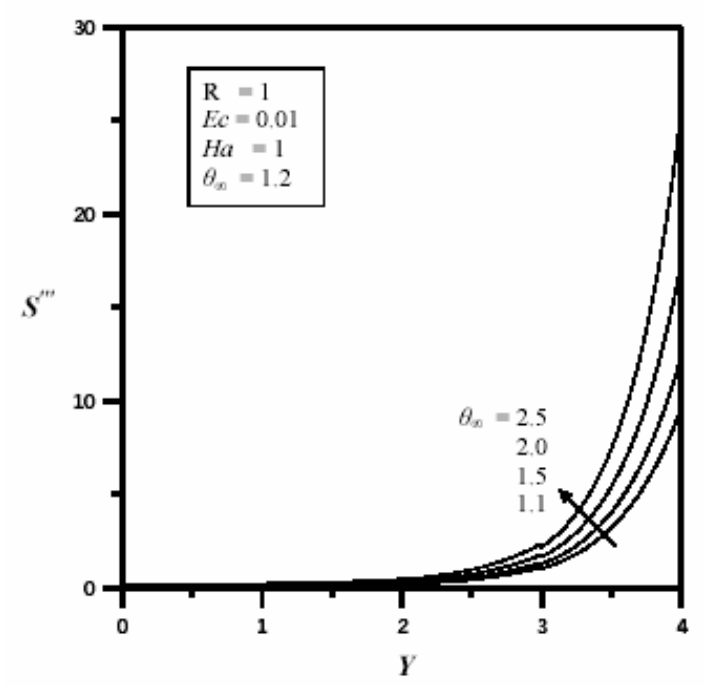

Figure 8. Dimensionless entropy generation for different values of $\left(\theta_{\infty}\right)$ at the end edge of the plate $(X=15)$.

\section{Concluding remarks}

This study was focused on the influence of transverse magnetic field effect on local entropy generation of steady two-dimensional laminar forced convection flow past a horizontal plate with a uniform stream parallel to its axis. The factors, which were found to affect the problem under consideration, are Hartmann number $(\mathrm{Ha})$, Eckert number $(E c)$, Prandtl number $(\mathrm{Pr})$, Joule heating parameter $(R)$ and the free stream temperature parameter $\left(\theta_{\infty}\right)$. It was found that, the volumetric entropy generation increased with increasing values of Hartmann number, Eckert number, and the Joule heating parameter. Whereas, the local entropy generation decreased as either of the free stream temperature parameter, and the Prandtl number increased. However, Joule heating parameter and Eckert number have dominating effect on local entropy generation than Hartmann number and Prandtl number.

\section{Nomenclature}

$B_{y} \quad$ magnetic field flux density

$C_{p} \quad$ specific heat of at constant pressure, $(\mathrm{kJ} / \mathrm{kg} . \mathrm{K})$

Ec Eckert Number

$g \quad$ gravitational acceleration, $\left(\mathrm{m} / \mathrm{s}^{2}\right)$

Ha Hartmann number

$k \quad$ thermal conductivity, (W/m.K)

Pr Prandtl number 


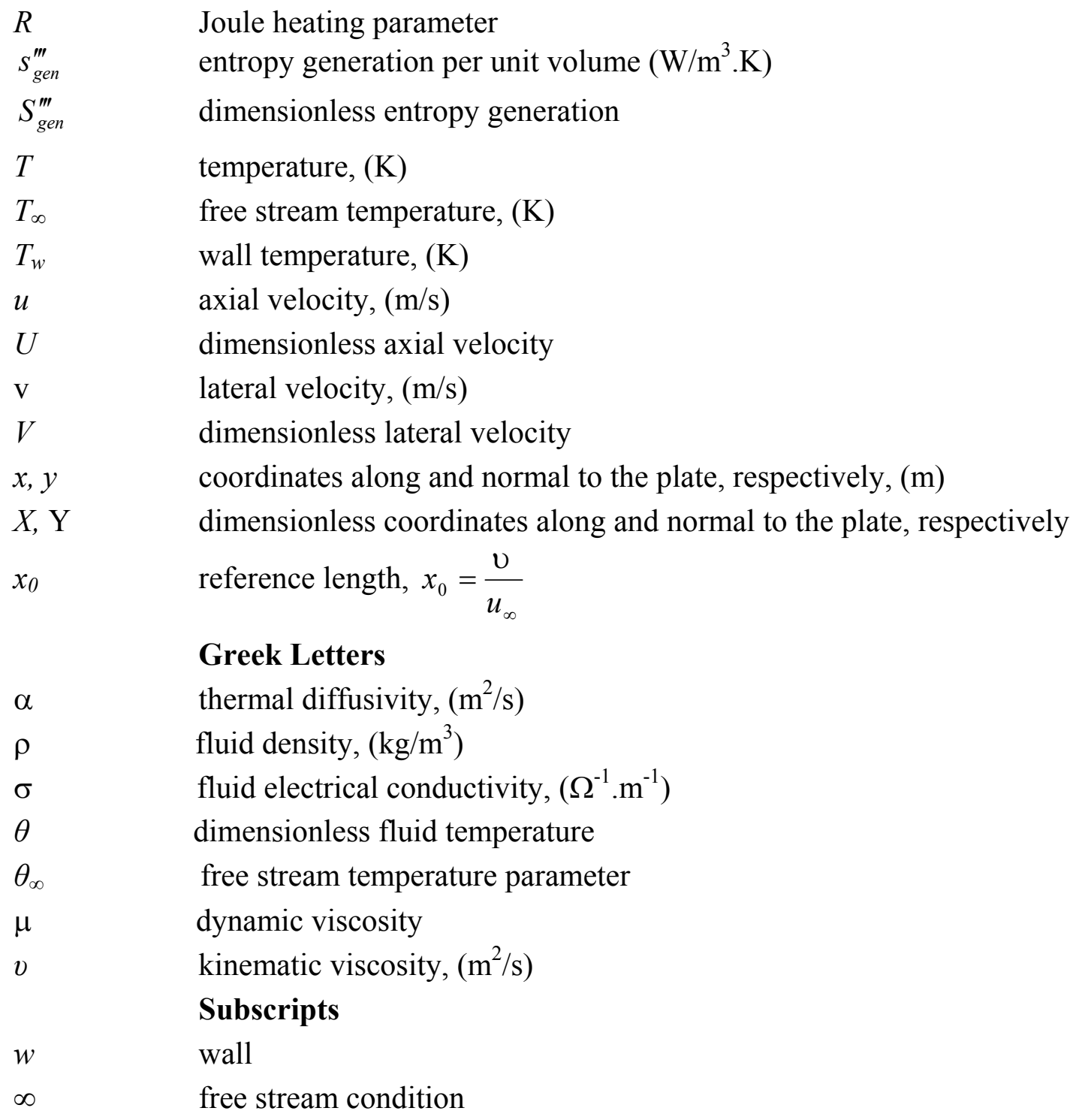

\section{References}

[1] Bejan, A., Second-law analysis in heat transfer and thermal design. Advanced heat transfer 1982, 15, 1-58.

[2] Bejan, A., Entropy Generation Minimization; CRC Press: New York, 1996.

[3] Abu-Hijleh B. A. K. and Heilen, W. N., entropy generation due to laminar natural convection over a heated rotating cylinder. Int. J. Heat and Mass Transfer 1999, 42, 4225-4233.

[4] Tasnim, S. H., Mahmud S. and Mamun, M. A. H., Entropy generation in a porous channel with hydromagnetic effect, Exergy, an Int. J. 2002, 2(4), 300-308.

[5] Mahmud, S. and Fraser R. A., The second law analysis in fundamental convective heat transfer problems, Int. J. of Therm. Sci. 2003, 42, 177-186. 
[6] Carrington, C. G. and Sun, Z. F., Second law analysis of combined heat and mass transfer in internal flow and external flows. Int. J. Heat and Fluid Flow. 1992, 132(1), 65-70.

[7] Arpaci, V.S. and Selamet A., Entropy production in boundary layers, J. Thermophys. Heat Transfer 1990, 4, 404-407.

[8] Khalkhali, H. Faghri, A. and Zuo, Z. J., Entropy generation in a heat pipe system, Applied Thermal Eng. 1999, 19(10), 1027-1043.

[9] Abu-Hijleh, B. A. K., entropy generation in laminar convection from an isothermal cylinder in cross flow, energy 1998, 23 (10), 851-857.

[10] Mahmud, S. and Fraser, R. A., Analysis of mixed convection-Radiation interaction in a vertical channel: Entropy generation, Exergy an Int. J. 2002, 2, 330-339.

[11] Haddad, O.M., Abu-Qudais, M., Abu-Hijleh, B.A., and Maqableh, A.M., Entropy generation due to laminar forced convection flow past a parabolic cylinder. Int. J. of Numerical Methods for Heat and Fluid Flow 2000, 10 (7), 770-779.

[12] Buhler, L., Laminar buoyant magnetohydrodynamics flow in a vertical rectangular ducts. Phys. Fluid 1998, 10, 223-236.

[13] Raptis, A. and Kafoussias, N., Heat transfer in flow through a porous medium bounded by an infinite vertical plane under the action of magnetic field. Energy Res. 1982, 6, 241-245.

[14] Chamkha, A. J. MHD free convection from a vertical plate in saturated porous medium. Appl. Math. Modeling 1997, 21, 603-609.

[15] Albshbeshy, E. M. A., Heat transfer over stretching surface with variable heat flux. J. Applied Phy. D 1998, 31, 19951-1954.

[16] Holman, J. P., Heat Transfer; $7^{\text {th }}$ ed., McGraw-Hill: New York, 1992; Chapter 12, pp 617623.

[17] Bejan, A. A., study of entropy generation in fundamental convective heat transfer. J. Heat Transfer 1979,101, 718-725.

[18] Patanker, S., Numerical Heat Transfer and Fluid Flow; Hemisphere: New York, USA, 1980.

[19] Kays, W. M. and Crawford, M.E, Convective Heat and Mass Transfer; McGraw-Hill: New York, USA, 1980.

(C) 2004 by MDPI (http://www.mdpi.org). Reproduction for noncommercial purposes permitted. 